\title{
Creutzfeldt-Jakob Disease or Herpes Encephalitis
}

R Ramirez ${ }^{1}$, J Márquez ${ }^{1}$, L Diaz², B Guillaumet ${ }^{3}$

\begin{abstract}
Creutzfeldt-Jakob disease (CJD) is a fatal rare disease which diagnosis is only confirmed by cerebral biopsy or demostrating prion transmissibility. Without these conditions, it is mandatory to rule of alternative diagnostics. Inside differential diagnosis acute herpes encephalitis must be considered, since its clinical presentation may resemble but its prognosis is considerably better. We present a 75 years old female with ataxia, cognitive impairments and seizures who had positive PCR for Herpes $\mathrm{I}$ in cerebrospinal fluid and positive Harrington's test too. We considered differential diagnosis between herpes encephalitis and CJD, despite Ig M for Herpes I was negative and neither MR nor electroencephalography showed characteristics of any of these diseases. With no response to antiviral treatment and demonstration of homocigousy for the methionin gen at 129 codon, the most probably diagnostic was CJD. Nevertheless, considering the different prognostic of both diseases brain biopsy was performed. It confirmed neuronal loss and spongiform degeneration with vacuolization with multiple PrP's deposits on immunohistochemistry. There were no signs of herpes encephalitis and Herpes virus PCR on brain biopsy was also negative. These results confirmed the diagnosis of CJD, sporadic variant. The patient died 3 months later.
\end{abstract}

Keywords: Creutzfeldt, encephalitis, herpes, Jakob

From: ${ }^{1}$ Internal Medicine, Hospital Sant Pau i Santa Tecla. Tarragona. Spain. ${ }^{2}$ Pathology, Hospital Universitari Joan XXIII Tarragona, Spain. ${ }^{3}$ Neurology, Hospital Sant Pau i Santa Tecla, Tarragona, Spain.

Correspondence: Dr R Ramirez, Montesinos Rambla Vella, nº 14 Tarragona 43003 (Spain) Fax: (34) 977234942, e-mail: rafaelramirezmontesinos@hotmail.com 


\section{INTRODUCTION}

Creutzfeldt-Jakob disease (CJD) is a rapidly progressive dementia due to prions. It is included in the group of "rare diseases" which require high suspicion index to be diagnosed. Definite diagnosis is only established either by specific neuropathologic pattern in brain biopsy or demonstrating transmissibility in animal models (1). Characteristic alterations in magnetic resonance $(\mathrm{MR})$, presence of the prionic protein $(\mathrm{PrP})$ in cerebrospinal fluid (CSF) and 129 codon mutation of prion protein gen can support the diagnosis. With them it is possible to establish a probability diagnosis in front of a typical picture of acute dementia, but it is necessary to rule alternative diagnostics. Acute infectious encephalitis can have a similar clinical presentation and must be considered in differential diagnosis. Herpes simplex virus type 1 encephalitis is the most frequent acute encephalitis in the world (2). It is diagnosed basically with typical clinical presentation and PCR for herpes virus in CSF. Characteristic MR alterations support the diagnosis. The case we submit reflects some diagnostic aspects to be considered in the differential diagnosis between both entities.

\section{CASE REPORT}

75 years old female with 2 months ataxia, cognitive impairments and seizures in the last 24 hours. Her relatives denied fever or headache. She had well controlled hypertension, diabetes, hiperlipidemia and hipothiroidism. Her habitual medication included levotiroxin 50 mcg, enalapril $20 \mathrm{mg}$, simvastatin $20 \mathrm{mg}$ and metformin 2 gr per day.

On physical examination she was stuporous, nonfebril and normotense, with Glasgow 11 (63-2), generalized hipertony, mioclonic movements and nistagmus. All reflexes were brisk and a left Babinsky was present. She had no meningeal signs. The rest of the exploration did not throw significant information. 
Blood test showed normal eritrosedimentation rate (ESR), liver, kidney, thyroid function, ionogram, and proteins electrophoresis. B vitamins, folic acid, and venous gasometry levels, were normal too. Tumor markers (alphafetoprotein, carcinoembrionic antigen, carcinoid antigens 19.9, 125 and 15.3, neuronal enolasa) calcitonin and HCG levels were no significant. Immunological study (including rheumatoid factor, antinuclear antigen, anti PR3, anti MPO, anti Ro, anti La, antimicrosomals, anticardiolipin, and antiperoxidasa antibodies) was normal, as well as ECA, RPR, cryoglobulins and cryoaglutinins. Serologic study for HIV, HBV, HCV, Rickettsia, Borrelia, Mycoplasma, Coxiella, Brucella, Adenovirus, Echovirus and Herpes simplex type II was negative. Herpes Simplex type I Ig G was positive (1/160), but Ig M title was not significant.

Thoracoabdominal and cranial tomography was normal, as well as cerebral MR with angio sequences. Electroencephalography showed a diffuse lentification with theta-delta waves alternation but no specific focalities or irritative paroxysms. In CSF analysis hiperproteinorraquia of 57, $9 \mathrm{mg} / \mathrm{dl}$, with 2 leukocytes for field and 620 erythrocytes for $\mathrm{mm}^{3}$ were detected. Glucose, ADA, VDRL, cultures, Ziehl-Neelsen and Pneumococcal antigen were negative. CSF pathological examination showed no alterations.

Study was extended with delta aminolevulinic acid, porphobilinogen, plumb and arsenic levels in $24 \mathrm{~h}$ urine, legionella antigenuria, cortisol, ceruloplasmin and paraneoplastic encephalitis (anti-Hu, anti amphiphysin and anti Ma-2) antibodies with negative results. Second lumbar puncture showed identical biochemical and microbiological parameters and negative ANAs, Ig G against Borrelia, immunoglobulin and oligoclonal bands quantification and polymerase chain reaction (PCR) for virus JC and Tropheryma Whipplei. Both PCR to simplex Herpes type I and Harrington's test (determination of prionic protein 14-3-3 for inmunoblot and colorimetry) were positive. 
Antiviral treatment with aciclovir was started, replaced by ganciclovir due to transient acute tubular necrosis. After 3 weeks of treatment there was no clinical response, with persistence of stupor and with generalized piramidalism, nistagmus and mioclonies. A second MR 10 weeks after the beginning of the symptoms was normal too. Polymorphism of 129 codon in CSF showing homocigousy for the methionin gen (129 M / M) was received, information compatible with the sporadic form of CJD. Brain biopsy was performed, confirming neuronal loss and spongiform degeneration with vacuolization (Figure 1) and multiple PrP's deposits on immunohistochemistry (Figure 2). No signs of herpes encephalitis were seen and herpes virus PCR on brain biopsy was negative. She died 3 months later. The autopsy was denied.

\section{DISCUSSION}

Creutzfeldt-Jakob disease is the most frequent disease produced by prions. Nevertheless, sporadic CJD, which is the most common form, occurs with a worldwide incidence of 1-1.5 per million (3). The majority of CJD cases correspond to sporadic mutations, although inside CJD spectrum familiar, iatrogenic or variant (also known vulgarly like "mad cows disease") can be distinguished. The paradigm of all of them is an acute dementia (alterations in conduct and memory, deficits of concentration and changes in the level of consciousness) accompanied by mioclonias (characteristically after startles) in $90 \%$ of cases, piramidalism, cerebellar alterations and nistagmus. Different phenotypes of the disease have been described depending on the genotype (homocigousy or heterocigousy) for methionin or valin in codon 129. The most frequent form of presentation, up to $70 \%$ of the cases, is MM1 or MV1 (Heidenhain myoclonic variant) genotype, habitual in old people and normally mortal in 3-4 months. MR showing bilateral high signal abnormalities in basal ganglia on diffusion- 
weighted imaging or fluid attenuated inversion recovery has $67 \%$ sensitivity and $93 \%$ specificity and support the diagnosis (4).

Ataxia, cognitive impairments and seizures can be present in herpes encephalitis. MR is the most sensitive and specific imaging method for HSV encephalitis, especially in the early course of the disease. Unilateral temporal lobe abnormalities on brain imaging are considered strong evidence for herpes simplex encephalitis (5). Besides that, MR may be normal in both CJD and acute herpes encephalitis. Ig M for Herpes I is normally positive in acute infection or reactivation but it presents huge individual variability (6). In this sense, negative titles cannot rule out this diagnostic therefore when herpes encephalitis is suspected PCR in CSF is mandatory, with $95 \%$ sensitivity and almost $100 \%$ specificity (5). In this case, despite positive PCR for Herpes I in CSF, PrP positivity and the absence of clinical response to antiviral treatment orientated to CJD. Polymorphism of 129 codon and brain biopsy confirmed CJD and rejected any alternative diagnostic.

The present case illustrates the difficulties that may be present in differential diagnosis between CJD and herpes encephalitis, with the necessity of brain biopsy to confirm CJD. Equally it reveals the caution with which we must interpret some complementary tests such as serology, PCR for herpes virus and MR in the differential diagnosis of similar cases. 


\section{REFERENCES}

1. cdc.gov [internet]. CDC's Diagnostic Criteria for Creutzfeldt-Jakob Disease (CJD), c2010 [updated 2015 February 11, cited 2015 July 9]. Available from: http://www.cdc.gov/prions/cjd/diagnostic-criteria.html

2. Chaudhuri A, Kennedy PGE. Diagnosis and treatment of viral encephalitis Postgrad Med J 2002; 78: 575-83.

3. Heinemann U, Krasnianski A, Meissner B, Varges D, Kallenberg K, Schulz-Schaeffer WJ, et al. Creutzfeldt-Jakob disease in Germany: a prospective 12-year surveillance. Brain 2007; 130: 1350-9.

4. Schröter A, Zerr I, Henkel K, Tschampa HJ, Finkenstaedt M, Poser S. Magnetic resonance imaging in the clinical diagnosis of Creutzfeld-Jakob disease. Arch Neurol 2000; 57: 1751-7.

5. McCabe K, Tyler K, Tanabe J. Diffusion-weighted MRI abnormalities as a clue to the diagnosis of herpes simplex encephalitis. Neurology 2003; 61: 1015-6.

6. Levitz RE. Herpes simplex encephalitis: a review. Heart Lung 1998; 27: 209-12. CJD or Herpes Encephalitis. 


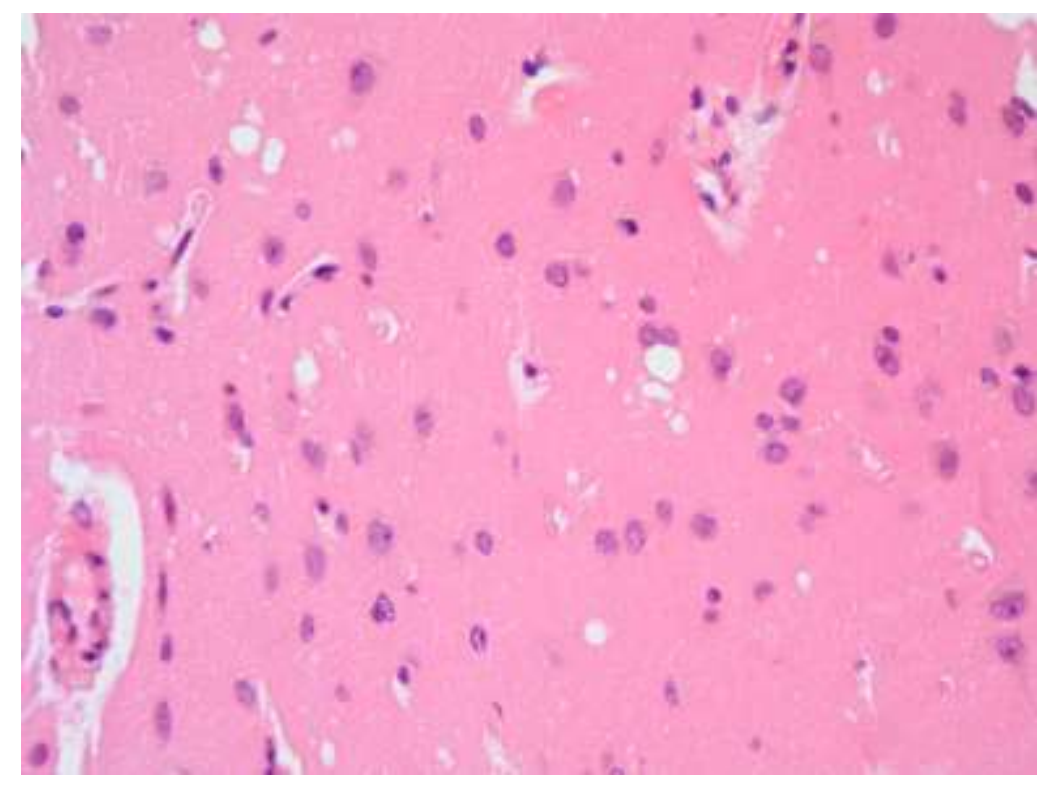

Fig. 1: Brain biopsy. Hematoxylin and Eosine. Neuronal loss and spongiform degeneration with vacuolization.

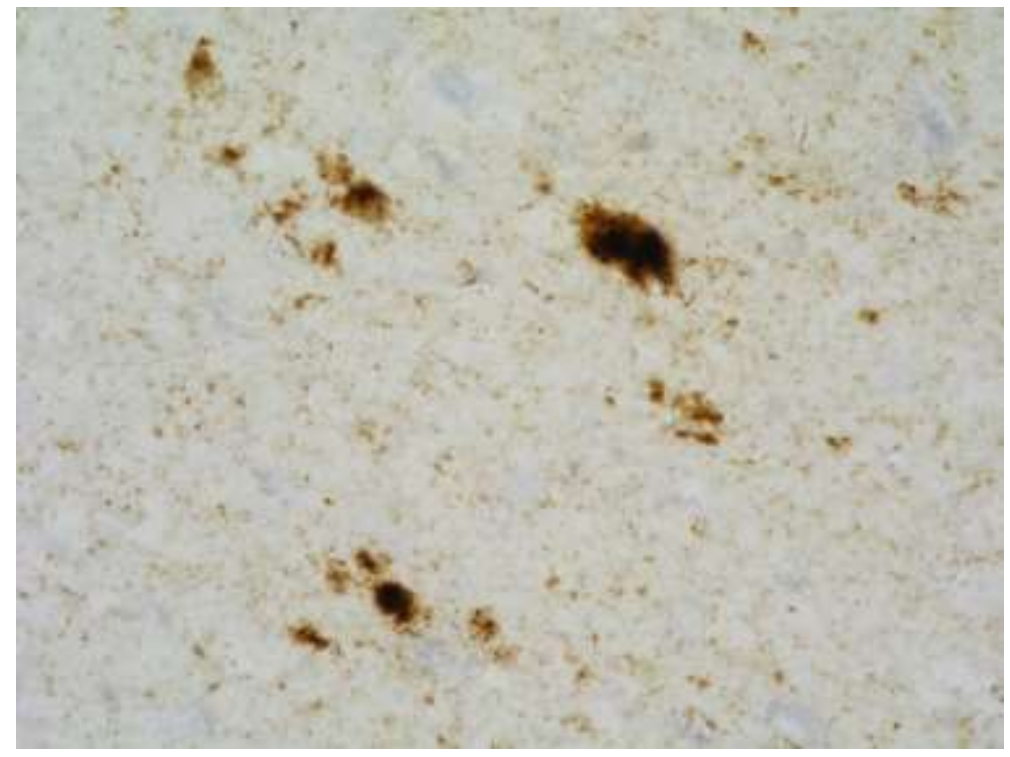

Fig. 2: Brain biopsy. Immunohistochemistry. Multiple PrP's deposits. 